\title{
CIBERCULTURA ESTUDIANTIL EN COMUNIDADES ACADÉMICAS DE UNIVERSIDADES PÚBLICAS MEXICANAS
}

\author{
José Refugio Romo-González*
}

Facultad de Filosofía y Letras. Universidad Autónoma de Chihuahua.

Javier Tarango**

Facultad de Filosofía y Letras. Universidad Autónoma de Chihuahua.

Patricia Murguía-Jáquez ${ }^{* * *}$

Facultad de Filosofía y Letras. Universidad Autónoma de Chihuahua.

$$
\text { Gerardo Ascencio-Baca**** }
$$

Facultad de Filosofía y Letras. Universidad Autónoma de Chihuahua.

\begin{abstract}
Resumen: Se presentan los resultados de una investigación tipo descriptiva, con características diagnósticas, sobre la medición de los niveles de cibercultura en dos comunidades estudiantiles de instituciones de educación superior, de carácter público, establecidas en la ciudad de Chihuahua, México (Facultad de Filosofía y Letras de la Universidad Autónoma de Chihuahua y Escuela Nacional de Antropología e Historia, Unidad Chihuahua). A través del uso de una metodología no experimental tipo transaccional-descriptiva y observacional, se evalúan cinco dimensiones o categorías conceptuales: 1) acceso, 2) uso, 3) apropiación tecnológica y social de las Tecnologías de la Información y las comunicaciones, 4) empoderamiento e 5) innovación social y desarrollo humano. Se concluye con la identificación de un conjunto de rasgos heterogéneos en el comportamiento de la cibercultura estudiantil y se agrega una serie de recomendaciones respecto al potencial por desarrollar en el análisis de un ámbito mayor de comunidades estudiantiles.
\end{abstract}

Palabras clave: Cibercultura; comunidades académicas; brecha digital; universidades públicas mexicanas.

Title: STUDENT' CYBERCULTURE IN ACADEMIC COMMUNITIES OF MEXICAN PUBLIC UNIVERSITIES.

Abstract: Presents the results of a descriptive research with diagnostic characteristics about the measurement of cyberculture levels in two student communities of higher education institutions, public, established in the city of Chihuahua, Mexico (Faculty of Philosophy and Letters of the Autonomous University of Chihuahua and the National School of Anthropology and History, in

\footnotetext{
*jromo@uach.mx

** jtarango@uach.mx

*** pmurguia@uach.mx

**** gbaca@uach.mx
}

Recibido: 19/10/2011; aceptado: 11/01/2012.

ROMO-GONZÁLEZ, J.R. et al. Cibercultura estudiantil en comunidades académicas de universidades públicas mexicanas. Anales de Documentación, 2012, vol. 15, nº 1. http://dx.doi.org/10.6018/analesdoc.15.1.138301. 
Chihuahua). Throughout the use of a non experimental methodology, transactionaldescriptive and observatory type evaluates five dimensions or conceptual categories: 1) approach, 2) usage and 3) technological and social appropriation of the communication and information technologies, 4) empowerment and 5) social innovation and human development. Concludes by identifying a set of heterogeneous features on the behavior of the student cyberculture and adds a number of advices regarding the potential to be developed in the analysis of a larger scope of student communities.

Keywords: Cyberculture; academic communities; digital divide; mexican public universities.

\section{INTRODUCCIÓN}

La educación superior en los países de economías emergentes, tales como aquellos que pertenecen a la región de Latinoamérica, especialmente México, demanda una adhesión marcada hacia las exigencias que delimita la sociedad de la información y el conocimiento, ya que al pretender ser entidades globalizadas, sus cambios en el desarrollo social, económico y político tendrán amplia vinculación con el acceso y uso adecuado de las tecnologías de la información y las comunicaciones (TIC), fenómeno que será manifiesto en la sociedad en general y para efectos de este trabajo, en las universidades públicas en particular.

Si bien, la atención a la población en general en cuestiones de acceso y uso de las TIC resulta compleja, se sabe que su abordaje dentro de medios controlados puede tener un efecto más concreto en la medición de indicadores de comportamiento, teniéndose así una comunidad educativa en la cual se logran medir sus avances concretos en diversos aspectos de carácter académico y, por tanto, se espera que se manifieste a corto plazo a través de la formación de ciudadanos con una cultura apropiada hacia el uso de los recursos electrónicos y digitales, quienes además consideran su servicio y contenidos en pro de una mayor productividad y competitividad (De Pablos Pons, 2010).

En el caso particular de México, se muestran evidentes atrasos en los indicadores de pertenencia a la sociedad de la información y el conocimiento especialmente en aspectos relacionados con la diferencia marcada en brecha digital, inclusión social y una inadecuada distribución social del conocimiento, situaciones que muestran una repercusión en la forma como se manifiesta el desarrollo social (Tarango y Lau, 2009). Además, deben considerarse aspectos culturales arraigados de la sociedad mexicana que denotan la existencia permanente de un sentido de trabajo individualista más que colectivista (Hofstede, 2001).

Es inminente que el cambio deberá ser generacional y en la medida que más sujetos tengan acceso al uso adecuado y productivo de las TIC, los rasgos culturales se generalizarán en mayor grado. La educación se considera un medio y momento peculiar de influir en comunidades estudiantiles precisas, esta vez de nivel superior, esperando que al egresar se conviertan en grupos de profesionales que incrustados en el ambiente laboral 
podrán contribuir de forma significativa en la sociedad, sin embargo, si su nivel de cibercultura es bajo durante su trayectoria estudiantil universitaria, las deficiencias se suponen serán evidentes.

La propuesta de este trabajo consistió en desarrollar un estudio diagnóstico, el cual permitió identificar las características ciberculturales con la detección de nivel en brecha digital de los sujetos estudiados y tener en consecuencia la posibilidad de aplicar medidas preventivas y correctivas con fines a mejorar el nivel de acceso y uso de las TIC, cuya consecuencia final sea impulsar la innovación social y el desarrollo humano de los egresados de las instituciones involucradas.

Las dos comunidades académicas que este estudio aborda ofrecen características peculiares, particularmente en la Universidad Autónoma de Chihuahua (UACH), que es la primera institución participante, posee diversas comunidades estudiantiles segmentadas principalmente por el área de conocimiento que cultivan y de las cuales se desconoce su nivel de cibercultura. Asimismo se ignora si existen brechas digitales en estas comunidades al ser comparadas con las de otras instituciones, ya sean públicas o privadas. Por lo que en principio se puede comenzar los estudios sobre cibercultura en la misma Universidad y específicamente en la Facultad de Filosofía y Letras (UACH-FFyL), comparándola con una comunidad similar en campos de conocimiento y en su carácter de institución pública, como lo es la Escuela Nacional de Antropología e Historia - Unidad Chihuahua (ENAH-Chihuahua), la cual se considera la segunda institución participante.

\section{FORMULACIÓN DEL PROBLEMA}

Al tratarse de una investigación de tipo descriptiva, a través de la cual se registran una serie de acontecimientos que permiten diagnosticar las condiciones de dos grupos de trabajo, así como desarrollar una comparación entre ambos comportamientos, el estudio parte del planteamiento de una serie de preguntas de investigación que se formulan a partir del problema anteriormente descrito dentro de la parte introductoria. Las preguntas son las siguientes:

a) ¿Se presenta una situación de igualdad $\mathrm{u}$ homogeneidad entre las distintas dimensiones de la cibercultura dentro de las comunidades estudiantiles de educación superior bajo análisis?

b) ¿Existen diferencias estadísticamente significativas entre las comunidades de la UACH-FFyL y la ENAH-Chihuahua, considerando cinco dimensiones de la cibercultura estudiantil?

c) ¿Existe una asociación escalonada entre las cinco dimensiones ciberculturales bajo estudio? Es decir, ¿un mayor acceso a las TIC se asocia con un nivel más alto de uso de las mismas?; ¿un mayor uso de las TIC, desencadena un mayor grado de apropiación tecnológica y social de ellas?; ¿una mayor apropiación de las TIC se asocia con un mayor 
empoderamiento de los individuos a través de las TIC?; y finalmente, ¿un mayor empoderamiento conduce a niveles más elevados de innovación social y desarrollo humano?

\section{OBJETIVOS DE LA INVESTIGACIÓN}

Los objetivos que se plantearon resolver esta investigación son los siguientes:

a) Caracterizar, de una manera descriptiva, la cibercultura estudiantil en dos comunidades educativas: la UACH-FFyL y la ENAH-Chihuahua, a través de cinco dimensiones o categorías conceptuales: 1) acceso, 2) uso, y 3) apropiación tecnológica y social de las TIC, 4) empoderamiento e 5) innovación social y desarrollo humano.

b) Establecer mediante un análisis diferencial, si las dos comunidades estudiantiles mantienen una diferencia estadísticamente significativa, llamada brecha digital, en lo general y en lo particular sobre cada una de las cinco dimensiones relativas a la cibercultura estudiantil.

\section{JUSTIFICACIÓN Y DELIMITACIÓN DEL PROBLEMA}

La presente investigación es de tipo descriptiva, debido a que su propósito principal es caracterizar o describir la cibercultura en comunidades estudiantiles de instituciones de educación superior y en segundo lugar, realizar un análisis diferencial entre comunidades estudiantiles participantes. Por tanto, puede decirse que el presente estudio es de carácter inicial y se circunscribe a dos comunidades estudiantiles en la ciudad de Chihuahua, México: la comunidad estudiantil de la UACH-FFyL y la ENAH-Chihuahua.

De hecho, su primer antecedente fue la definición de una metodología precisa para el desarrollo de esta clase de estudios, identificando los principales elementos metodológicos que debe comprender el abordaje de estudios de cibercultura en comunidades estudiantiles, dicha propuesta fue publicada por Hélice: Revista Venezolana de Ciencias de la Información (vol., 2, $\mathrm{n}^{\mathrm{o}}$ 2, agosto-diciembre, 2010) bajo el título: Elementos para el estudio de la cibercultura estudiantil en comunidades educativas (Tarango, Romo y Murguía, 2010).

En la práctica, este tipo de investigaciones se justifican en el hecho de que a través de la misma se obtendrá una caracterización de la cibercultura y las brechas digitales que se presentan entre las comunidades estudiantiles de nivel superior, comparadas con otras comunidades estudiantiles provenientes de otras instituciones, ya sean públicas o privadas, con la finalidad expresa de poder plantear posteriormente las estrategias y las acciones necesarias para reducir dichas brechas digitales y contribuir para que los estudiantes mejoren su nivel de cultura digital, orientándolos hacia una cibercultura con altos niveles de empoderamiento que conlleven a la innovación social y desarrollo humano y con ello 
contribuir a elevar la calidad de vida de la sociedad en general y en particular de su propia comunidad estudiantil.

\section{MARCO TEÓRICO CONCEPTUAL}

Actualmente la sociedad está viviendo una etapa de transición que lo está transformando todo de un modo extraordinariamente acelerado. Se está siendo protagonistas del paso de la sociedad industrial a la sociedad de la información y el conocimiento, se está abriendo paso un nuevo paradigma y se abandonan conceptos y modelos tradicionales para dar cabida a un nuevo orden (Cervantes Galván, 2007). Ante esta nueva situación, se pueden hacer dos reflexiones, una a nivel global y otra a nivel del entorno mexicano. A nivel global se puede decir que la desigualdad en el mundo está aumentando de forma espectacular, generando graves tensiones y conflictos, por lo que se debe luchar por disminuir estas brechas. A escala nacional, es imprescindible emplear el máximo de esfuerzos a todos los niveles para consolidar la posición de México en acciones modernas, de la tecnología y la innovación (Aguiar, 2007). De lo contrario, el crecimiento constante del déficit tecnológico relegará a México a una posición de debilidad frente a los países pioneros de la nueva sociedad de la información y el conocimiento.

En los elementos estructurales y funcionales de la sociedad de la información, según Martin (1988) y Webster (2002), es posible distinguir cinco elementos estructurales que describen lo que es una sociedad son: tecnológico, económico, ocupacional, elemento tiempo-espacio y cultural. Es la combinación de estos cinco elementos lo que puede caracterizar el que una sociedad específica, haya entrado o no a la era de la información, donde obviamente, los países más ricos y desarrollados llevan la delantera.

Como ya se mencionó anteriormente, uno de los elementos estructurales y funcionales de la sociedad de la información es la cultura o los factores culturales, por lo que en este apartado se revisa el concepto de "cibercultura" que engloba precisamente a este elemento funcional de la sociedad de la información. El término de "cibercultura" en general se refiere a la cultura nacida de la aplicación de las nuevas TIC en medios de comunicación como el Internet (Avogadro, 2007). Sin embargo se puede hablar no sólo de Internet sino de un espacio más amplio y variado como el "ciberespacio", el cual puede acotarse como un nuevo medio de comunicación que surge de la interconexión mundial de los sistemas de datos. Es decir el ciberespacio incluye la infraestructura material de la información digital y el universo de informaciones que contiene, donde el Internet apenas es una parte, tal vez la más importante pero no la única.

Lévy (2007) extiende el concepto de cibercultura y lo entiende como la cultura propia de las sociedades en cuyo seno, las tecnologías digitales configuran decisivamente las formas dominantes tanto de información, comunicación y conocimiento como de investigación, producción, organización y administración. Es decir, en la cibercultura, además de sistemas materiales y simbólicos, están integrados agentes y prácticas 
culturales, interacciones y comunicaciones, colectivos, instituciones y sistemas organizativos, una multiplicidad de contenidos y representaciones simbólicas junto con valores, significados, interpretaciones, legitimaciones, etc.

Rueda Ortiz (2008) considera a la cibercultura como un campo de estudio a partir del cual es posible comprender las transformaciones culturales ligadas a la introducción de tecnologías digitales en las sociedades contemporáneas a través de relaciones complejas de entramados tecnosociales, en tres ámbitos: el ejercicio del poder, la acción social colectiva y la experiencia estética. Castell (2001), desde una óptica sociológica identifica cuatro niveles en la cibercultura: la cultura tecnomeritocrática (basada en la fe y en la bondad inherente al desarrollo científico y tecnológico como componentes clave en el proceso de desarrollo de la humanidad); la cultura de los hackers (surge de las redes de programadores interconectados en línea y colaborando en proyectos autodefinidos de programación creativa); la cultura de las comunidades virtuales (inicialmente vinculada a los movimientos contraculturales, caracterizada por la comunicación libre y horizontal); y la cultura empresarial, que ha surgido en torno a los usos comerciales del Internet y otros medios digitales). Tubella y Vilaseca (2008), por otro lado introducen la discusión del determinismo tecnológico en el caso de las TIC en contrastación con la democratización de las nuevas tecnologías y plantean la necesidad de un modelo de pluralismo cultural capaz de garantizar que los intercambios entre las culturas no supongan la asimilación y la disolución de las comunidades culturales más desfavorecidas, reclamando la adopción y el perfeccionamiento de sistemas democráticos de control de su utilización social, a fin de que respondan al interés general y vayan en beneficio del conjunto de la humanidad. El mismo Lévy (2007) al percibir diversos grados de cibercultura, acuña el término de "ciberculturización" como el proceso general de cambio en las formas de comunicación en las distintas comunidades bajo estudio, introduciendo la posibilidad de establecer niveles o estadios de cultura digital.

Otros autores, tales como Castell (2001), Trejo Delarbre (2006), Ríos (2006) y Morales Campos (2006), han estudiado los diversos grados de ciberculturización bajo la denominación de estudios sobre la brecha digital, entendiéndola como aquella diferencia entre las personas que utilizan las nuevas TIC y aquellas que no tienen acceso a las mismas. El término "brecha digital" también hace referencia a las diferencias que hay entre grupos según su capacidad para utilizar las TIC de forma eficaz, debido a los distintos niveles de alfabetización y capacidad tecnológica, sin embargo no se llega al nivel de señalar las diferencias entre aquellos grupos que tienen acceso a contenidos digitales de calidad y aquellos que no lo tienen. Específicamente Ríos (2006), efectúa un estudio sobre la brecha digital entre estudiantes de escuelas públicas y privadas en Venezuela y sus posibles repercusiones en la incorporación a la sociedad de la información y el conocimiento. 


\section{MODELO CONCEPTUAL SIMPLIFICADO}

El modelo conceptual simplificado que se asume en este trabajo, establece de manera básica las relaciones que se supone entre las cinco dimensiones conceptuales que conforman la cibercultura estudiantil. Es decir, las cinco dimensiones estarían concatenadas y asociadas de manera escalonada, donde la dimensión más básica impulsa el nivel de la dimensión superior y así sucesivamente.

En este caso, el modelo conceptual asumido establece que un mayor acceso a las TIC se asocia con un nivel más alto de uso de las mismas. A su vez, un mayor uso de las TIC, desencadena un mayor grado de apropiación tecnológica y social de ellas y una mayor apropiación de las TIC se asocia con un mayor empoderamiento de los individuos a través de las TIC, considerando finalmente que un mayor empoderamiento conduce a niveles más elevados de innovación social y desarrollo humano. Esta descripción es representada gráficamente a través de la Figura 1.

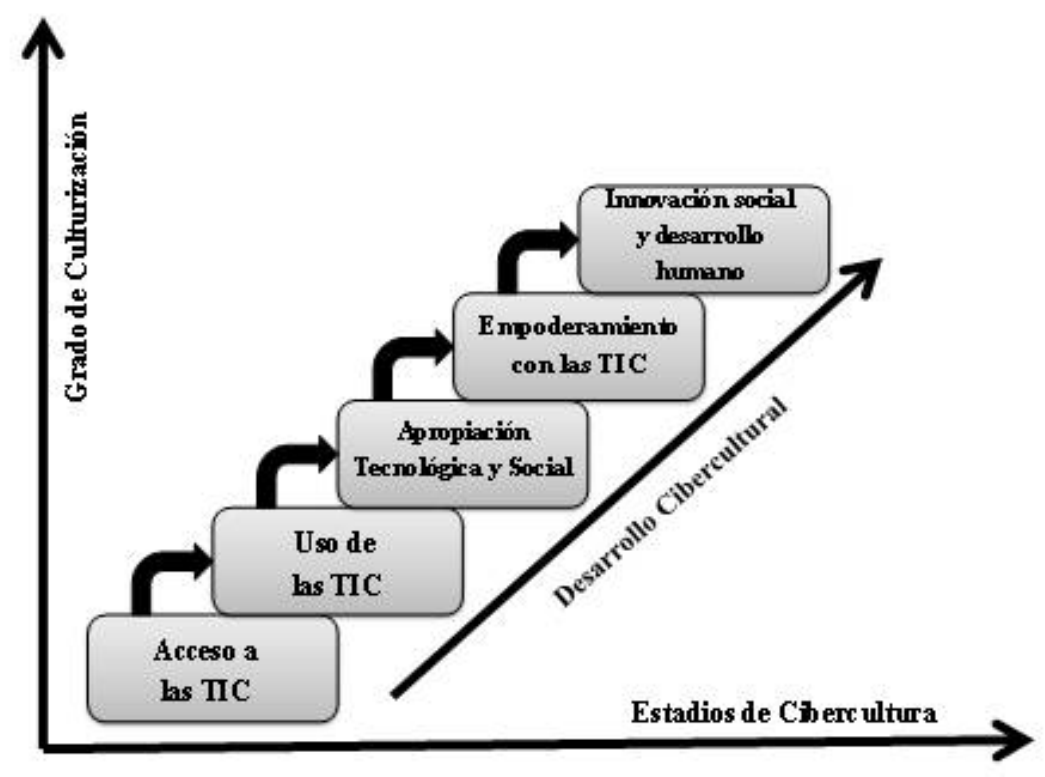

Figura 1. Modelo conceptual simplificado.

\section{INFORMACIÓN REQUERIDA}

Se consideran cinco apartados básicos en las necesidades específicas de información, uno por cada dimensión cibercultural, además del apartado de datos generales del estudiante o unidad de análisis. Las cinco dimensiones de estudio de la cibercultura 
muestran una derivación de datos específicos (cuatro renglones por cada uno), ambos niveles de requerimientos de información son representados en el Cuadro I.

\begin{tabular}{|c|c|}
\hline Dimensión de cibercultura & Información específica \\
\hline 1) Acceso a las TIC & $\begin{array}{l}\text { - Acceso a telefonía celular } \\
\text { - Acceso a Internet } \\
\text { - Acceso a intranets } \\
\text { - Acceso a conexiones inalámbricas }\end{array}$ \\
\hline 2) Uso de las TIC & $\begin{array}{l}\text { - Uso básico del celular } \\
\text { - Uso avanzado del celular } \\
\text { - Uso básico del Internet } \\
\text { - Uso avanzado del Internet } \\
\end{array}$ \\
\hline $\begin{array}{l}\text { 3) Apropiación tecnológica y } \\
\text { social de las TIC }\end{array}$ & $\begin{array}{l}\text { - Compartición informal de información } \\
\text { - Participación en discusiones/debates } \\
\text { virtuales } \\
\text { - Uso de Internet para aprendizaje } \\
\text { - Participación en comunidades virtuales } \\
\text { con metas sociales, políticas, culturales o } \\
\text { económicas }\end{array}$ \\
\hline 4) Empoderamiento & $\begin{array}{l}\text { - Internet como medio de cambio social } \\
\text { - Internet y participación ciudadana } \\
\text { - Internet para disminuir distancia con } \\
\text { autoridades } \\
\text { - Internet para participar en toma de } \\
\text { decisiones }\end{array}$ \\
\hline $\begin{array}{l}\text { 5) Innovación social y desarrollo } \\
\text { humano }\end{array}$ & $\begin{array}{l}\text { - Intervención de Internet en problemas } \\
\text { comunitarios } \\
\text { - Aportación de nuevas ideas y soluciones } \\
\text { - Apoyos a grupos sociales vulnerables } \\
\text { - Promoción de la justicia, democracia y } \\
\text { desarrollo humano por medio del Internet }\end{array}$ \\
\hline $\begin{array}{l}\text { 6) Datos generales de los } \\
\text { estudiantes }\end{array}$ & $\begin{array}{l}\text { - Datos escolares } \\
\text { - Datos socioeconómicos } \\
\text { - Datos de acceso a TIC } \\
\text { - Comentarios personales }\end{array}$ \\
\hline
\end{tabular}

Cuadro I. Dimensiones de cibercultura y necesidades específicas de información.

\section{PLANTEAMIENTO DE LA HIPÓTESIS}

Se plantean tres hipótesis básicas o principales:

a) "Las cinco dimensiones de la cibercultura en las comunidades bajo análisis, muestran una situación heterogénea" 
b) "Las comunidades estudiantiles bajo estudio presentan diferencias significativas en cuanto a cibercultura, es decir existen brechas digitales entre las mismas"

c) "Existe una asociación escalonada entre las cinco dimensiones ciberculturales bajo estudio"

\section{METODOLOGÍA}

Por sus características, la investigación es de carácter no experimental, es decir de tipo observacional, donde los hechos ya están dados y sin ningún control o manipulación sobre el entorno. Se adopta un diseño no experimental transaccional descriptivo con un solo corte en el tiempo: Diciembre 2010 - Marzo 2011, para poder cubrir los objetivos del trabajo.

La población de interés la constituyen dos comunidades estudiantiles de instituciones de educación superior de la ciudad de Chihuahua, México, para lo cual se realizó un muestreo tipo probabilístico y aleatorio, aunque posteriormente con los datos característicos de los encuestados se estratificaron y validó la muestra. La unidad de análisis está constituida por un estudiante perteneciente a las comunidades bajo estudio, sin ninguna restricción de edad, sexo, nivel socioeconómico, carrera que cursa o nivel académico.

La definición del tamaño de la muestra, al emplear los estimadores, probabilidad de ocurrencia $\mathrm{p}=75 \%$ (Porcentaje de estudiantes con empoderamiento) y probabilidad de no ocurrencia $\mathrm{q}=25 \%$ (Porcentaje de estudiantes sin empoderamiento); un error estándar del $5 \%$ y un nivel de confianza del $90 \%$, resulta un tamaño de la muestra (n) de 203 estudiantes de licenciatura de las comunidades estudiantiles UACH-FFyL y de la ENAHChihuahua. Pero al considerar el tamaño de la población objetivo en la UACH-FFyL que es de 693 estudiantes de licenciatura, el tamaño muestral queda ajustado a 157 estudiantes de la UAH-FFyL y con una población objetivo de 76 estudiantes de la comunidad estudiantil ENAH-Chihuahua (en donde se ofrece únicamente el programa de Licenciatura en Antropología Social), el tamaño muestral queda ajustado a 55 estudiantes. Es decir entre ambas comunidades se recolectó información de 212 estudiantes. Las poblaciones de ambas comunidades estudiantiles corresponden a agosto de 2009.

La muestra aleatoria, en el caso de la comunidad estudiantil UACH-FFyL, dado que está compuesta por diversos programas educativos, deberá conservar una estructura aproximadamente igual a la poblacional, para lo cual se vigiló el cumplimiento de la distribución muestral por carrera profesional, tal como se expresa en el Cuadro II. 


\begin{tabular}{lccc}
\hline Programa de Licenciatura & $\begin{array}{c}\text { Número } \\
\text { de estudiantes }\end{array}$ & $\begin{array}{c}\text { Carga } \\
\text { muestral }\end{array}$ & $\begin{array}{c}\text { Tamaño de la } \\
\text { muestra }\end{array}$ \\
\hline Filosofía & 102 & $15 \%$ & 23 \\
Letras Españolas & 170 & $24 \%$ & 38 \\
Lengua Inglesa & 165 & $24 \%$ & 38 \\
Ciencias de la Información & 187 & $27 \%$ & 42 \\
Historia & 26 & $4 \%$ & 7 \\
Periodismo & 43 & $6 \%$ & 9 \\
\hline TOTAL & $\mathbf{6 9 3}$ & $\mathbf{1 0 0 \%}$ & $\mathbf{1 5 7}$ \\
\hline
\end{tabular}

Cuadro II. Muestra aleatoria: Comunidad estudiantil UACH-FFyL.

La selección de la muestra fue completamente aleatoria, empleando como marco muestral los listados oficiales de alumnos inscritos en el semestre con el que se efectuó el trabajo de campo, proporcionados por las autoridades académicas de las propias instituciones.

Respecto a la recolección de datos, el instrumento seleccionado para la medición de las distintas variables es el cuestionario, por considerarse el procedimiento más adecuado para el caso. El cuestionario incluye las secciones o apartados correspondientes con las necesidades de información ya planteadas anteriormente, su carácter es anónimo y auto administrado personalmente (está compuesto por 20 preguntas en total, correspondiendo cuatro preguntas por cada una de las cinco dimensiones de cibercultura evaluadas, ver Anexo 1). La confiabilidad y validez del cuestionario aplicado, se consignan en el apartado de resultados de este trabajo. La codificación de los datos recolectados fue elaborada sobre todas las preguntas o equivalentes no precodificados, elaborando computacionalmente el respectivo cuaderno de códigos.

El almacenamiento físico de los datos fue hecho en medios magnéticos y con respaldo duro en papel, utilizando software de aplicación, específicamente el paquete estadístico SPSS para Windows versión 15. Se aplicaron los tipos de análisis estadísticos apropiados para estudiar los datos, de acuerdo a la naturaleza del trabajo y los niveles de medición de las variables, así como de los objetivos de la investigación. De acuerdo a lo anterior se plantean diversos tipos de análisis, los mismos que se resumen en el cuadro III de este documento. 


\begin{tabular}{ll}
\hline Objetivos & Tipos de análisis \\
\hline Caracterización descriptiva de la cibercultura & Análisis frecuencial y descriptivo \\
en comunidades estudiantiles a través de & \\
cinco dimensiones o categorías conceptuales. & \\
\hline Establecer si las comunidades estudiantiles & Prueba de hipótesis sobre medias \\
bajo estudio mantienen una diferencia & Análisis de correlación \\
estadísticamente significativa en cibercultura, & Análisis de varianza \\
tanto en lo general como en lo particular & \\
sobre cada una de las cinco dimensiones & \\
relativas a la cibercultura.
\end{tabular}

Cuadro III. Tipos de análisis estadísticos utilizados.

\section{ANÁLISIS DE RESULTADOS}

El estudio partió con la aplicación de una prueba piloto, que se ejecutó a 11 estudiantes de la comunidad estudiantil de la ENAH-Chihuahua que cursaban el tercer semestre de licenciatura que accedieron a participar en la prueba. Al término de la aplicación se abrió un espacio de tiempo para discutir dudas sobre redacción, secuencia y lógica de las preguntas, así como sobre el formato de presentación del cuestionario, indicándose que no hubo grandes alteraciones sobre la propuesta original.

Previo a la aplicación de la prueba piloto se realizó un análisis de validez de la escala que se emplearía para observar y medir el concepto más importante dentro de este trabajo, es decir la cibercultura estudiantil. Para ello, se sometió la validación del contenido del instrumento de medición a una ronda o interacciones de cuatro expertos en el tema, utilizando el método Delphi de análisis y evaluación de información cualitativa (Landeta, 2005).

En cuanto al análisis de confiabilidad, el cual permitió determinar el grado en que los elementos del cuestionario se relacionan entre sí, obtener un índice global de la replicabilidad o de la consistencia interna de la escala en su conjunto e identificar elementos problemáticos que deben ser excluidos de la escala, se utilizó la prueba Alfa de Cronbach, la cual arrojó, de acuerdo a los casos de items estudiados, un total de casos válidos de $9(81.8 \%)$, excluidos $2(18.2 \%)$ de un total de $11(100 \%)$. Los datos estadísticos de fiabilidad según la prueba Alfa de Cronbach resultó de 0.751 y superior a 0.70 , por lo que la escala de confiabilidad de la escala es aceptable y por lo tanto el error de medición que provocaría la aplicación de la escala es de un $24.9 \%$.

$\mathrm{Al}$ asumir un modelo lineal aditivo para el constructo de interés fundamental en esta investigación (cibercultura estudiantil), el cálculo del nivel respectivo para el constructo deberá hacerse sumando los puntajes correspondientes a todas las variables incluidas en las cinco dimensiones del constructo. El modelo lineal aditivo propuesto para la dimensión o constructo bajo análisis es: 


\section{Cibercultura Estudiantil $=$ f (Acceso a las TIC + Uso de las TIC + Apropiación tecnológica y social de las TIC + Empoderamiento + Innovación social y desarrollo} humano)

Como en el constructo de cibercultura estudiantil se incluyeron 20 variables, quedaría:

$$
\text { Cibercultura Estudiantil }=X_{1.1}+X_{1.2}+X_{1.3}+X_{1.4}+X_{2.1}{ }^{+} \ldots+X_{5.3}+X_{5.4}
$$

Donde cada una de las cinco dimensiones tiene cuatro variables, haciendo de esa forma el total de las 20 variables ya mencionadas.

Con la finalidad de comprobar el supuesto de aditividad en el modelo propuesto, se aplicó la Prueba de Aditividad de Tukey (también llamada prueba de no aditividad por otros autores) y los resultados fueron: casos válidos 163 (90.6\%), casos excluidos 17 $(9.4 \%)$ de un total de $180(100 \%)$.

Otras características muestrales, ya sean frecuenciales o descriptivas, que permiten conocer a mayor profundidad la naturaleza de la muestra empleada para estudiar la cibercultura estudiantil de las dos comunidades bajo análisis es representado a través del análisis de los datos generales que se recolectaron a través de la encuesta. El reporte se realizó en el mismo formato empleado en el instrumento de medición, consignando los promedios cuando la variable es cuantitativa y los porcentajes cuando es cualitativa (se incluyen los resultados principales a los propósitos de este documento): promedio escolar en el semestre anterior 84 sobre 100; edad promedio 21.9 años; género: $37 \%$ masculino, $63 \%$ femenino; estado civil $91 \%$ soltero y $9 \%$ casado; actualmente trabajan: $36 \%$ si y $64 \%$ no; promedio de computadoras por casa: 1.8 ; Internet en casa: $78 \%$ si poseen y $22 \%$ no; promedio de teléfonos celulares por casa: 3.6; y, lugar en que con mayor frecuencia se conectan a Internet: $26 \%$ en la escuela, $68 \%$ en el hogar y $6 \%$ en ciber-oficina.

El nivel de cibercultura estudiantil estimado sobre la muestra de estudiantes, arrojó un promedio, calculado con la media aritmética, de 68.36 puntos con una desviación estándar de 12.228 puntos con un rango de 67 puntos, donde el estudiante con menor cibercultura obtuvo 27 puntos y el de mayor cibercultura 94 puntos sobre un total posible de 100 puntos. La distribución probabilística de la variable es la distribución normal, según la prueba de Kolmogorov - Smirnov mostró una sig=0.320. Los resultados generales del análisis de las cinco dimensiones de la cibercultura estudiantil se resumen en la Figura 2.

En los resultados generales, es decir sin considerar el tipo de institución de que se trate y abarcando las cinco dimensiones de la cibercultura, se aprecia que la dimensión de uso de las TIC presenta el promedio más alto, incluso por encima del acceso a las TIC, lo cual indica que la comunidad estudiantil en general, se encuentra apenas en un nivel de desarrollo cibercultural de uso y no de apropiación tecnológica y social. Por otro lado, los promedios de empoderamiento e innovación social y desarrollo humano resultaron 
superiores a los de apropiación tecnológica y social, lo cual no concuerda con la teoría, debiendo haber resultado con promedios inferiores. Esto puede entenderse como un fenómeno especial donde antes de saturar un nivel de cibercultura se pasa al siguiente, donde con pocos recursos ciberculturales se introducen innovaciones sociales y desarrollo humano, que sería la etapa superior de la cibercultura.

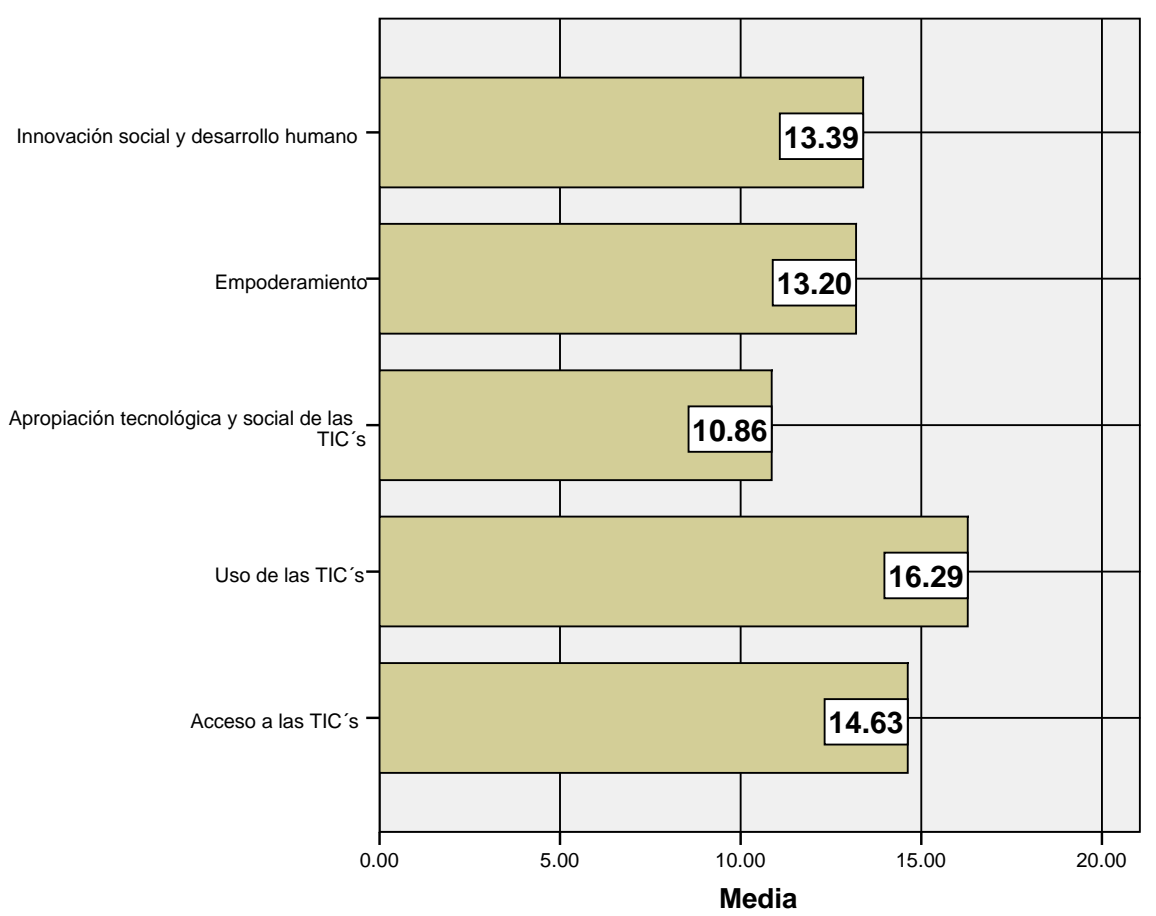

Figura 2. Resultados generales de las cinco dimensiones de cibercultura estudiantil.

Al realizar un análisis de correlación entre las cinco dimensiones ciberculturales, se aprecian relaciones significativas escalonadas, lo cual concuerda con la teoría cibercultural, es decir el acceso a las TIC está asociado con su uso $(\mathrm{p}=0.000)$, apropiación $(\mathrm{p}=0.009)$ y empoderamiento $(\mathrm{p}=0.000)$; no así con la innovación social y desarrollo humano, donde las relaciones con el acceso a las TIC no resultaron ser significativas $(p=0.255)$.

Similarmente, el uso de las TIC se asocia con su apropiación tecnológica y social, así como con el empoderamiento e innovación social y desarrollo humano, siendo significativas estas tres asociaciones. Finalmente, el empoderamiento se asocia significativamente con la innovación social y el desarrollo humano $(\mathrm{p}=0.000)$, tal como se muestra en el Cuadro IV. 


\begin{tabular}{|c|c|c|c|c|c|c|}
\hline & & $\begin{array}{l}\text { Acceso a } \\
\text { las TIC's }\end{array}$ & $\begin{array}{l}\text { Uso de } \\
\text { las TIC's }\end{array}$ & $\begin{array}{l}\text { Apropiación } \\
\text { tecnológica y } \\
\text { social de las } \\
\text { TIC's }\end{array}$ & $\begin{array}{l}\text { Empoder } \\
\text { amiento }\end{array}$ & $\begin{array}{l}\text { Innovación } \\
\text { social y } \\
\text { desarrollo } \\
\text { humano } \\
\end{array}$ \\
\hline \multirow[t]{3}{*}{ Acceso a las TIC's } & Correlación de Pearson & 1 & $.348^{* *}$ & $.197^{\star \star *}$ & $.270^{\star \star}$ & .087 \\
\hline & Sig. (bilateral) & & .000 & .009 & .000 & .255 \\
\hline & $\mathrm{N}$ & 174 & 173 & 174 & 168 & 171 \\
\hline \multirow[t]{3}{*}{ Uso de las TIC's } & Correlación de Pearson & $.348^{\star *}$ & 1 & $.293^{\star \star}$ & $.304^{\star \star *}$ & $.168^{\star}$ \\
\hline & Sig. (bilateral) & .000 & & .000 & .000 & .025 \\
\hline & $\mathrm{N}$ & 173 & 179 & 179 & 173 & 176 \\
\hline \multirow{3}{*}{$\begin{array}{l}\text { Apropiación tecnológica } \\
\text { y social de las TIC's }\end{array}$} & Correlación de Pearson & $.197^{* *}$ & $.293^{* *}$ & 1 & $.261^{\star *}$ & $.154^{*}$ \\
\hline & Sig. (bilateral) & .009 & .000 & & .001 & .041 \\
\hline & $\mathrm{N}$ & 174 & 179 & 180 & 174 & 177 \\
\hline \multirow[t]{3}{*}{ Empoderamiento } & Correlación de Pearson & $.270^{* \star \star}$ & $.304^{\star \star}$ & $.261^{* *}$ & 1 & $.622^{\star \star}$ \\
\hline & Sig. (bilateral) & .000 & .000 & .001 & & .000 \\
\hline & $\mathrm{N}$ & 168 & 173 & 174 & 174 & 171 \\
\hline \multirow{3}{*}{$\begin{array}{l}\text { Innovación social y } \\
\text { desarrollo humano }\end{array}$} & Correlación de Pearson & .087 & $.168^{*}$ & $.154^{*}$ & $.622^{\star *}$ & 1 \\
\hline & Sig. (bilateral) & .255 & .025 & .041 & .000 & \\
\hline & $\mathrm{N}$ & 171 & 176 & 177 & 171 & 177 \\
\hline
\end{tabular}

**. La correlación es significativa al nivel 0,01 (bilateral).

*. La correlación es significante al nivel 0,05 (bilateral).

Cuadro IV. Correlaciones entre dimensiones de cibercultura.

Todas las asociaciones resultaron positivas, es decir a mayor puntuación de una dimensión básica, resultan mayores puntuaciones de las dimensiones superiores. Por ejemplo, a mayor acceso a las TIC, mayor uso y apropiación de ellas; a mayor apropiación de las TIC, mayor empoderamiento; etc.

Los niveles o grados de asociación aunque significativos resultaron bajos, con la notoria excepción de la relación entre empoderamiento e innovación social y desarrollo humano, donde el coeficiente de correlación de Pearson resultó de 0.622 , lo cual indica un nivel de asociación "alto". Es decir, el nivel de empoderamiento cibercultural conlleva a mayores grados de innovación social y desarrollo humano en el ciberespacio, datos que se pueden proyectar a campos reales tanto de carácter social como económico, político y cultural. El diagrama de dispersión de esta asociación se muestra en la Figura 3, en donde de acuerdo al diagrama de dispersión, se aprecia claramente que a mayor empoderamiento cibercultural, mayores niveles de innovación social y desarrollo humano. 


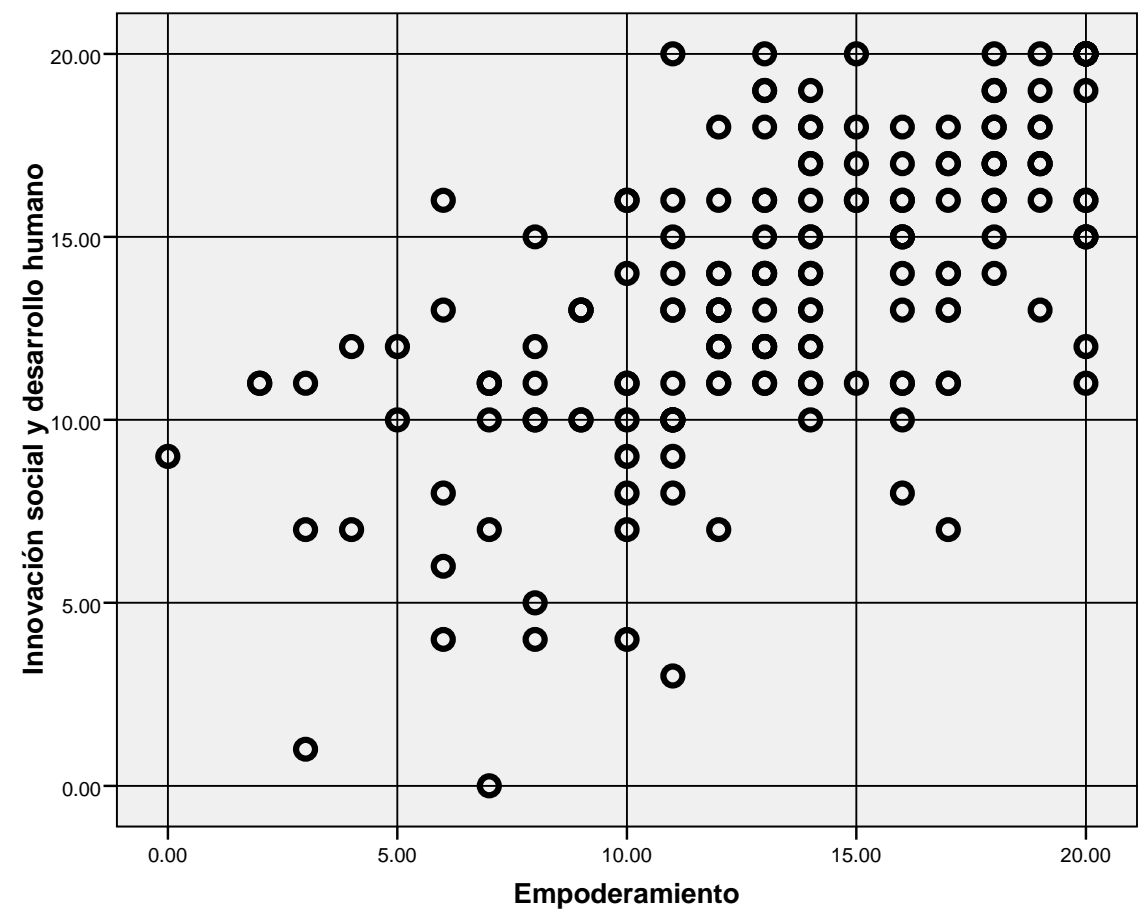

Figura 3. Asociación entre empoderamiento e innovación social y desarrollo humano.

Respecto a los resultados diferenciales por comunidad estudiantil, es decir, se desarrolla un análisis comparativo, distinguiendo entre las comunidades estudiantiles de UACH-FFYL y ENAH-Chihuahua, tal como se expresa en el Cuadro V.

\begin{tabular}{lcc}
\hline $\begin{array}{l}\text { Dimensiones de la cibercultura } \\
\text { estudiantil }\end{array}$ & UACH-FFYL & $\begin{array}{c}\text { ENAH- } \\
\text { Chihuahua }\end{array}$ \\
\hline 1. Acceso a las TIC & 14.75 & 14.02 \\
2. Uso de las TIC & 16.60 & 14.88 \\
3. Apropiación Tecnológica y Social de las & & \\
TIC & 10.69 & 10.70 \\
4. Empoderamiento & 13.38 & 12.40 \\
5. Innovación Social y Desarrollo Humano & 13.51 & 13.50 \\
\hline \multicolumn{1}{c}{ Cibercultura general } & $\mathbf{6 8 . 6 9}$ & $\mathbf{6 7 . 2 1}$ \\
\hline
\end{tabular}

Cuadro V. Cibercultura en comunidades estudiantiles.

En los resultados se aprecian niveles más altos de acceso y uso de las TIC en la comunidad estudiantil de la UACH-FFYL que en la ENAH-Chihuahua y sin embargo, tienen el mismo nivel de apropiación tecnológica y social de las TIC. La comunidad 
UACH-FFYL tiene a su vez un nivel más alto de empoderamiento con las TIC, pero a pesar de ello arroja el mismo nivel de innovación social y desarrollo humano que la comunidad ENAH-Chihuahua, lo cual quiere decir que se están subutilizando en la comunidad estudiantil de la UACH-FFYL las dimensiones de acceso, uso y apropiación tecnológica y social de las TIC, ya que debería arrojar en consonancia mejores niveles de innovación social y desarrollo humano. Esto también pudiera explicarse por un mayor nivel de concientización social por parte de los miembros de la comunidad estudiantil de antropología.

En cuanto al promedio general de cibercultura estudiantil, la comunidad UACH-FFyL arrojó un mayor nivel (68.69) que la comunidad ENAH-Chihuahua (67.21). No obstante, al realizar las respectivas pruebas de hipótesis para las dos muestras independientes y las dimensiones respectivas, incluyendo la cibercultura general promedio, no se encontraron diferencias estadísticas significativas, excepto para la dimensión de uso de las TIC, donde la comunidad UACH-FFyL arrojó una media (16.60 puntos) significativamente mayor $(\mathrm{p}=0.003)$ a la de la ENAH-Chihuahua (14.88 puntos).

Al extender el análisis diferencial de la cibercultura estudiantil a las carreras involucradas dentro de las dos comunidades estudiantiles, resultó ser la Licenciatura en Lengua Inglesa la carrera con el mayor nivel de cibercultura, seguida por Filosofía, Ciencias de la Información y Antropología Social, siendo Historia y Letras Españolas las carreras con los niveles más bajos en cibercultura, tal como lo muestra en la Figura 4. 


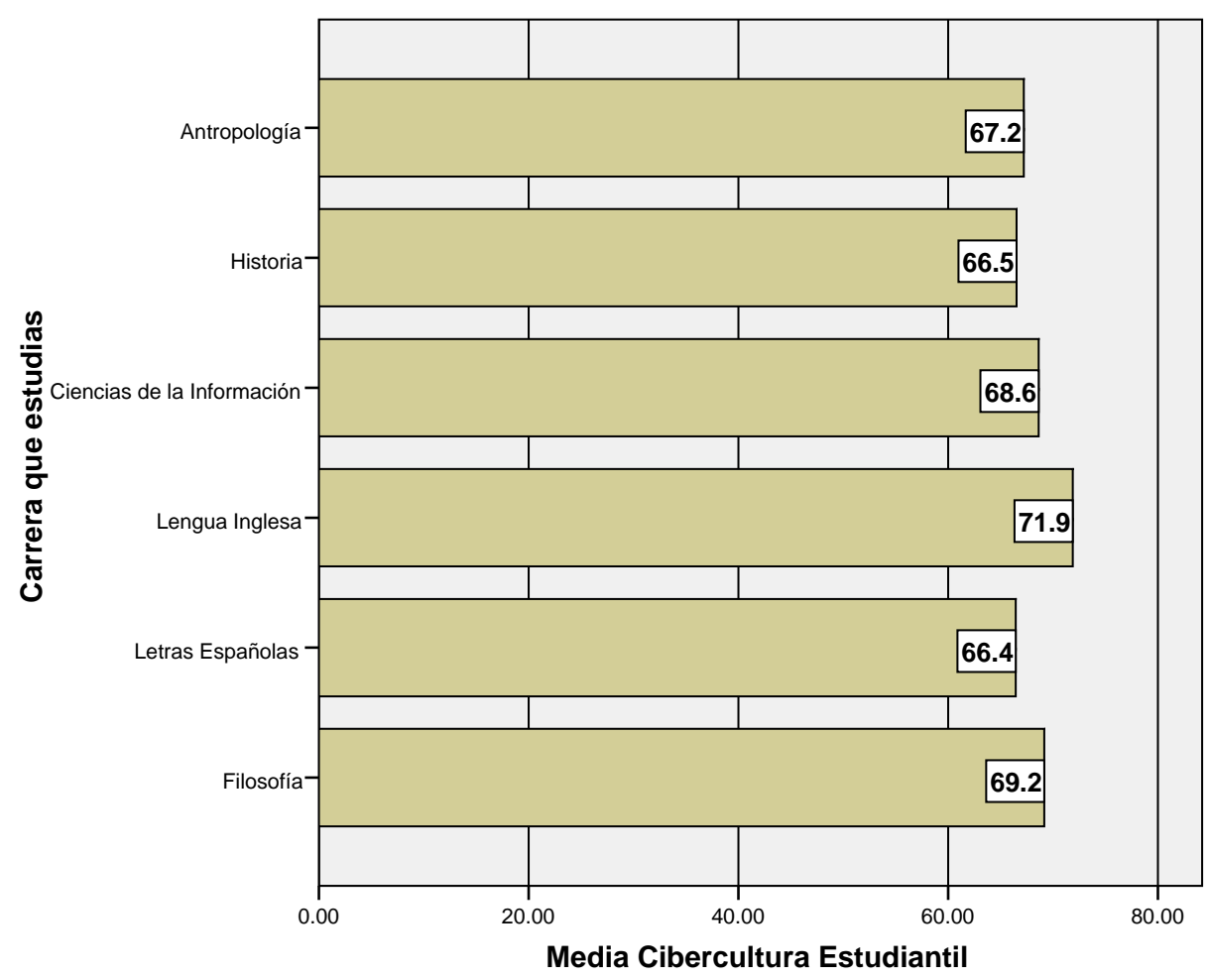

Figura 4. Cibercultura estudiantil por carrera.

Sin embargo, al realizar un Análisis de varianza de un Factor para detectar diferencias significativas entre las medias de las carreras, no se detectaron diferencias significativas $(\mathrm{p}=0.550)$, por lo cual se concluye que las medias en cibercultura por carrera son iguales, tal como se representa en el cuadro de Análisis de varianza identificado como Cuadro VI.

\begin{tabular}{|l|r|r|r|r|r|}
\hline & $\begin{array}{c}\text { Suma de } \\
\text { cuadrados }\end{array}$ & gl & $\begin{array}{c}\text { Media } \\
\text { cuadrática }\end{array}$ & \multicolumn{1}{c|}{$\mathrm{F}$} & Sig. \\
\hline Inter-grupos & 602.956 & 5 & 120.591 & .802 & .550 \\
Intra-grupos & 23770.818 & 158 & 150.448 & & \\
Total & 24373.774 & 163 & & & \\
\hline
\end{tabular}

Cuadro VI. Análisis de varianza entre carreras profesionales.

Además, se desarrolló un análisis diferencial por carrera profesional y dimensión cibercultural, a través del cual se permitió detectar diferencias no sólo entre carreras profesionales en sí, sino en su diferenciación con las dimensiones ciberculturales (Figura 5), observándose que la carrera con mayor acceso y uso de TIC, así como con mayor 
empoderamiento fue Lengua Inglesa, sin embargo aunque con menores niveles de acceso y uso de TIC la carrera de Filosofía mostró los niveles más altos en innovación social y desarrollo humano, mientras que Antropología Social, mostró la mejor apropiación tecnológica y social de las TIC. Es decir que el simple acceso y uso de las TIC no basta para que posteriormente se presente la apropiación social de la tecnología y su empoderamiento y que finalmente se alcancen altos niveles de innovación social y desarrollo humano. La orientación que tienen las carreras de Filosofía y Antropología Social, hacen que sus estudiantes usen y apliquen las TIC con mayor sentido social y de desarrollo humano.

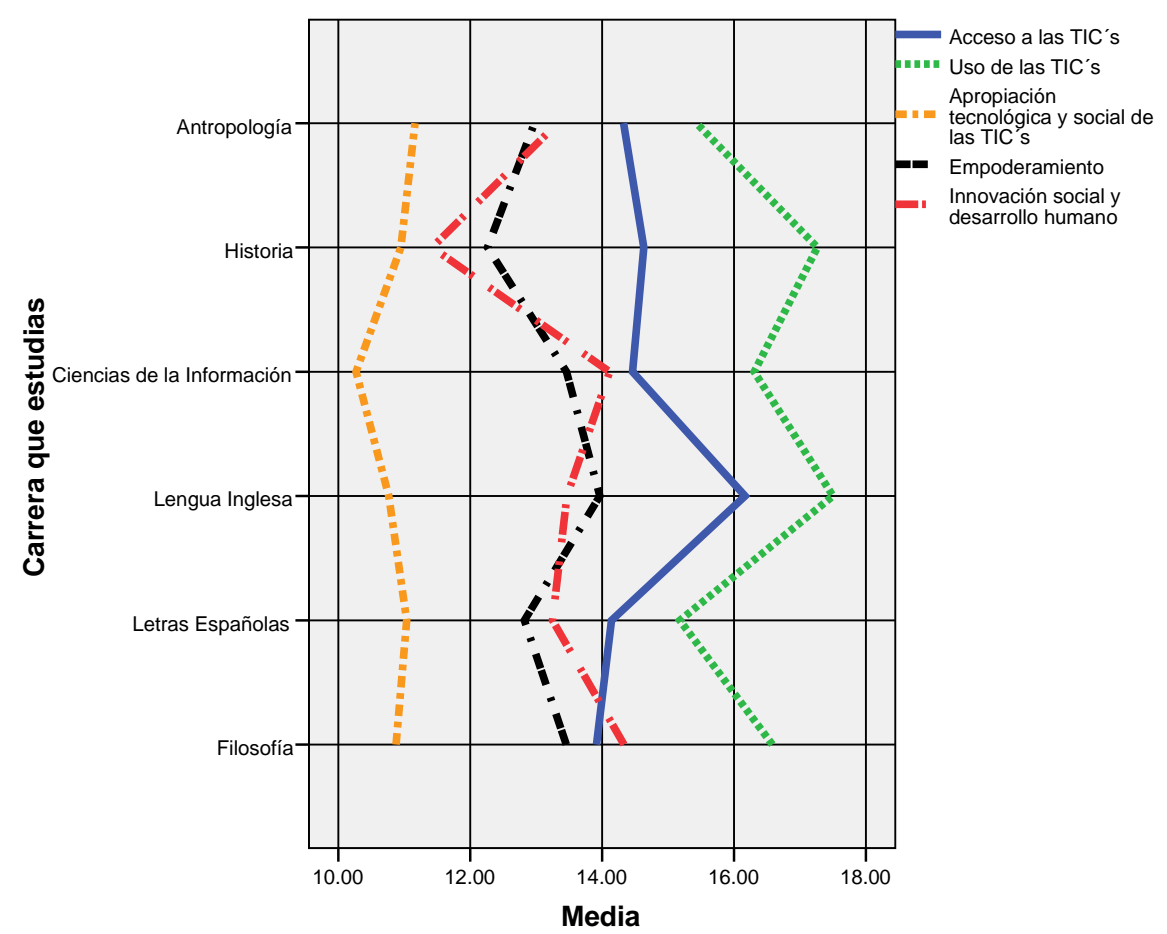

Figura 5. Análisis diferencial por carrera profesional y dimensión cibercultural.

Lo anterior queda corroborado en la carrera de Ciencias de la Información, la cual tiene buenos niveles de acceso y uso de las TIC, pero apunta el nivel más bajo en cuanto apropiación tecnológica y social de las mismas TIC, así como bajos niveles de innovación social y desarrollo humano. Sin embargo, al ejecutar el Análisis de varianza de un Factor para las cinco dimensiones de la cibercultura sobre las seis carreras, sólo se detectaron diferencias estadísticas significativas en las medias poblacionales para la dimensión 
cibercultural de uso de las TIC ( $\mathrm{p}=0.004)$, según lo muestra el Cuadro VII de Análisis de varianza.

\begin{tabular}{|c|c|c|c|c|c|c|}
\hline & & $\begin{array}{c}\text { Suma de } \\
\text { cuadrados }\end{array}$ & $\mathrm{gl}$ & $\begin{array}{c}\text { Media } \\
\text { cuadrática }\end{array}$ & $\mathrm{F}$ & Sig. \\
\hline \multirow[t]{3}{*}{ Acceso a las TIC's } & Inter-grupos & 105.719 & 5 & 21.144 & \multirow[t]{3}{*}{1.702} & \multirow[t]{3}{*}{.137} \\
\hline & Intra-grupos & 2086.954 & 168 & 12.422 & & \\
\hline & Total & 2192.672 & 173 & & & \\
\hline \multirow[t]{3}{*}{ Uso de las TIC's } & Inter-grupos & 193.829 & 5 & 38.766 & \multirow[t]{3}{*}{3.653} & \multirow[t]{3}{*}{.004} \\
\hline & Intra-grupos & 1835.802 & 173 & 10.612 & & \\
\hline & Total & 2029.631 & 178 & & & \\
\hline \multirow{3}{*}{$\begin{array}{l}\text { Apropiación tecnológica } \\
\text { y social de las TIC's }\end{array}$} & Inter-grupos & 8.608 & 5 & 1.722 & \multirow[t]{3}{*}{121} & \multirow[t]{3}{*}{.988} \\
\hline & Intra-grupos & 2479.586 & 174 & 14.250 & & \\
\hline & Total & 2488.194 & 179 & & & \\
\hline \multirow[t]{3}{*}{ Empoderamiento } & Inter-grupos & 83.408 & 5 & 16.682 & \multirow[t]{3}{*}{.775} & \multirow[t]{3}{*}{.569} \\
\hline & Intra-grupos & 3616.706 & 168 & 21.528 & & \\
\hline & Total & 3700.115 & 173 & & & \\
\hline \multirow{3}{*}{$\begin{array}{l}\text { Innovación social y } \\
\text { desarrollo humano }\end{array}$} & Inter-grupos & 128.863 & 5 & 25.773 & \multirow[t]{3}{*}{1.593} & \multirow[t]{3}{*}{.165} \\
\hline & Intra-grupos & 2767.351 & 171 & 16.183 & & \\
\hline & Total & 2896.215 & 176 & & & \\
\hline
\end{tabular}

Cuadro VII. Análisis de varianza de las cinco dimensiones de cibercultura.

La prueba de separación de medias de Tukey, arrojó específicamente diferencias significativas en el uso de las TIC entre las carreras de Lengua Inglesa y Antropología Social, siendo la primera la que observó la media más alta; detectando asimismo diferencias significativas en innovación social y desarrollo humano entre las carreras de Filosofía e Historia, siendo Filosofía la carrera con más alto nivel en innovación social y desarrollo humano, tal como se logra observar en el Cuadro VIII sobre la prueba de Tukey sobre uso de las TIC y en el Cuadro IX sobre innovación social y desarrollo humano. 


\begin{tabular}{|c|c|c|c|}
\hline \multirow[b]{2}{*}{ Carrera que estudias } & \multirow[b]{2}{*}{$\mathrm{N}$} & \multicolumn{2}{|c|}{$\begin{array}{c}\text { Subconjunto para alfa } \\
=.05\end{array}$} \\
\hline & & 1 & 2 \\
\hline Antropología & 45 & 14.8889 & \\
\hline Letras Españolas & 30 & 15.1667 & 15.1667 \\
\hline $\begin{array}{l}\text { Ciencias de la } \\
\text { Información }\end{array}$ & 30 & 16.6333 & 16.6333 \\
\hline Filosofía & 25 & 16.7200 & 16.7200 \\
\hline Historia & 19 & 17.2632 & 17.2632 \\
\hline Lengua Inglesa & 30 & & 17.5000 \\
\hline Sig. & & .076 & .085 \\
\hline
\end{tabular}

Se muestran las medias para los grupos en los subconjuntos homogéneos.

a. Usa el tamaño muestral de la media armónica $=27$. 926.

b. Los tamaños de los grupos no son iguales. Se utilizará la media armónica de los tamaños de los grupos. Los niveles de error de tipo I no están garantizados.

Cuadro VIII. Prueba Tukey de uso de las TIC.

\section{CONCLUSIONES}

De acuerdo al análisis de datos presentados y según los planteamientos hipotéticos que son el punto central de este estudio, se concluye lo siguiente:

1. Las cinco dimensiones de la cibercultura en las comunidades estudiantiles bajo análisis (UACH-FFYL y ENAH-Chihuahua), mostraron una situación heterogénea, rechazando por lo tanto, la hipótesis nula de homogeneidad entre las distintas dimensiones.

2. De manera general, es decir sin considerar el tipo de comunidad estudiantil de procedencia, se encontró que la dimensión de uso de las TIC presenta el promedio más alto, incluso por encima del acceso a las TIC, lo cual indica que la comunidad estudiantil en su conjunto se encuentra apenas en un nivel de desarrollo cibercultural de uso y no de apropiación tecnológica y social.

3. Los promedios de empoderamiento e innovación social y desarrollo humano resultaron superiores a los de apropiación tecnológica y social, lo cual no concuerda con la teoría, debiendo haber resultado con promedios inferiores, 
pudiendo entenderse esto como un fenómeno especial donde antes de saturar un nivel de cibercultura se pasa al siguiente, donde con pocos recursos ciberculturales se introducen innovaciones sociales y desarrollo humano, que sería la etapa superior de la cibercultura, atribuyendo esto al grado de conciencia social que mantienen las comunidades estudiantiles bajo análisis.

4. Las asociaciones entre las dimensiones ciberculturales resultaron significativas y escalonadas aunque con niveles bajos de correlación, lo cual corrobora la hipótesis de asociación escalonada planteada en este estudio, con la notoria excepción de la relación entre empoderamiento e innovación social y desarrollo humano, donde el nivel de la correlación resultó alto, siendo ello un claro indicador de que el empoderamiento cibercultural conlleva a mayores grados de innovación social y desarrollo humano, que se pueden proyectar a campos reales tanto de carácter social como económico, político y cultural.

\begin{tabular}{|l|r|r|r|}
\hline & \multirow{2}{*}{ Carrera que estudias } & $\mathrm{N}$ & \multicolumn{2}{|c|}{$\begin{array}{c}\text { Subconjunto para alfa } \\
=.05\end{array}$} \\
\cline { 3 - 4 } & 19 & 11.4211 & \multicolumn{1}{c|}{2} \\
\hline Historia & 30 & 13.3000 & 13.3000 \\
Letras Españolas & 30 & 13.4667 & 13.4667 \\
Lengua Inglesa & 44 & 13.5000 & 13.5000 \\
Antropología & 29 & 14.2759 & 14.2759 \\
Ciencias de la & & & 14.5600 \\
Información & 25 & & .852 \\
Filosofía & & .093 & \\
Sig. & & & \\
\hline
\end{tabular}

Se muestran las medias para los grupos en los subconjuntos homogéneos.

a. Usa el tamaño muestral de la media armónica $=27$. 713.

b. Los tamaños de los grupos no son iguales. Se utilizará la media armónica de los tamaños de los grupos. Los niveles de error de tipo I no están garantizados.

\section{Cuadro IX. Prueba Tukey sobre innovación social y desarrollo humano.}

5. Se detectaron niveles más altos de acceso y uso de las TIC en la comunidad estudiantil de la UACH-FFYL que en la ENAH-Chihuahua, teniendo sin embargo el mismo nivel de apropiación tecnológica y social de las TIC.

6. La comunidad UACH-FFYL tiene un nivel más alto de empoderamiento con las TIC, pero a pesar de ello arroja el mismo nivel de innovación social y desarrollo 
humano que la comunidad ENAH-Chihuahua, lo cual quiere decir que se están subutilizando en la UACH-FFYL las dimensiones de acceso, uso y apropiación tecnológica y social de las TIC, ya que debería arrojar en consonancia mejores niveles de innovación social y desarrollo humano. Esto también pudiera explicarse por un mayor nivel de concientización social por parte de los miembros de la comunidad estudiantil de ENAH-Chihuahua.

7. Al extender el análisis diferencial de la cibercultura estudiantil a las carreras involucradas dentro de las dos comunidades estudiantiles, resultó ser Lengua Inglesa la carrera con el mayor nivel de cibercultura, seguida por Filosofía, Ciencias de la Información y Antropología Social, siendo Historia y Letras Españolas las carreras con los niveles más bajos en cibercultura.

8. La carrera con mayor acceso y uso de TIC, así como mayor empoderamiento fue Lengua Inglesa, sin embargo aunque con menores niveles de acceso y uso de TIC la carrera de Filosofía mostró los niveles más altos en innovación social y desarrollo humano, mientras que Antropología mostró la mejor apropiación tecnológica y social de las TIC.

9. Por lo anterior, se puede concluir que el simple acceso y uso de las TIC no basta para que posteriormente se presente la apropiación social de la tecnología y su empoderamiento y que finalmente se alcancen altos niveles de innovación social y desarrollo humano. La orientación que tienen las carreras de Filosofía y Antropología, hacen que sus estudiantes usen y apliquen las TIC con mayor sentido social y de desarrollo humano.

10. Lo anterior queda corroborado en la carrera de Ciencias de la Información, la cual tiene buenos niveles de acceso y uso de las TIC, pero apunta el nivel más bajo en cuanto apropiación tecnológica y social de las mismas TIC, así como bajos niveles de innovación social y desarrollo humano.

\section{RECOMENDACIONES}

Tal como se menciona dentro de la justificación del presente trabajo, en donde se especifica que se tiene como antecedente la identificación de una metodología precisa para el estudio de cibercultura en comunidades estudiantiles y el desarrollo del presente estudio aplicado en dos entidades, se proponen las siguientes recomendaciones para la continuidad del trabajo investigativo:

1. Extender el trabajo de investigación hacia otras áreas académicas de la UACH para caracterizar la cibercultura en otras comunidades estudiantiles y poder establecer si existen brechas digitales motivadas por este factor.

2. Extender la investigación hacia comunidades estudiantiles adscritas a instituciones privadas, para analizar las posibles brechas digitales entre 
estudiantes de escuelas públicas vs privadas, como un posible segundo factor de influencia.

3. Una vez extendida la investigación y teniendo una caracterización detallada de las brechas en cultura digital entre las diversas comunidades estudiantiles, se recomienda la elaboración de un plan general de culturización digital a nivel estudiantil dentro de las instituciones participantes, que permita además de acortar las brechas digitales, orientar la cultura digital hacia altos niveles de empoderamiento que conlleven a la innovación social y desarrollo humano y con ello elevar la calidad de vida de la sociedad en general.

\section{BIBLIOGRAFÍA}

AGUILAR, H. El futuro no espera. Políticas para desarrollar la sociedad del conocimiento. Buenos Aires, Argentina: La Crujía Ediciones, 2007.

AVOGADRO, M. Glosario de nuevas tecnologías de la información y la comunicación. Razón y Palabra, 2007, $\mathrm{n}^{\circ} 55$.

CASTELLS, M. La era de la información. Economía, sociedad y cultura. Vol. 1, 2 y 3. México, D.F.: Siglo Veintiuno, 2001.

CERVANTES GALVÁN, E. La sociedad del conocimiento. Oportunidades y estrategias. México, D.F.: Trillas, 2007.

DE PABLOS PONS, J. Universidad y sociedad del conocimiento: Las competencias informacionales y digitales. En: Competencias informacionales y digitales en Educación Superior/ Universitat Oberta de Catalunya, RUSC, 2010, vol. 7, nº 2, p. 616.

LANDETA, J. El método Delphi. (2a ed.). Barcelona, España: Airel, 2002.

LÉVY, P. Cibercultura. La cultura de la sociedad digital. Antropos. México, D.F.: Universidad Autónoma Metropolitana, 2010.

MARTIN, W. J. The information society - idea or entity? Aslib Proceeding, 1998, vol. $40, n^{\circ} 11 / 12$, p. 303-309.

MORALES CAMPOS, E. Infodiversidad y cibercultura: globalización e información en América Latina. Buenos Aires, Argentina: Alfagrama, 2006.

RÍOS, R. Brecha digital entre estudiantes de escuelas públicas y privadas. Telematique, 2006, vol. 5, n 2. Universidad Rafael Bellosos Chacin. Zulia, Venezuela.

RUEDA ORTIZ, R. Cibercultura: metáforas, prácticas sociales y colectivos en red. Nómadas, 2008, $\mathrm{n}^{\circ}$ 28, Abril: 8-20. Universidad Central. Medellín, Colombia.

TARANGO, J. y LAU, J. Brecha más cognitiva que digital: papel de las habilidades informativas en países emergentes. Ponencia presentada en la II Conferencia Internacional sobre Brecha Digital e Inclusión Social, Universidad Carlos III de Madrid, Leganés, Madrid, 28-30 octubre, 2009.

.; ROMO, J.R. y MURGUÍA, P. Elementos para el estudio de la cibercultura estudiantil en comunidades educativas $=$ Elements for the approach of students cyberculture into academic communities. Hélice: Revista Venezolana de Ciencias de la Información, agosto-diciembre 2010, vol. 2, nº 2 . 
TREJO DELARBRE, R. Viviendo en el Aleph: La sociedad de la información y sus laberintos. Barcelona, España: Gedisa Editorial, 2006.

TUBELLA I CASADEVALL, I. y VILASECA I REQUENA, J. Sociedad del Conocimiento. Universitat Oberta de Catalunya. Barcelona, España: Editorial UOC, 2005.

WEBSTER, F. Theories of the Information Society. $2^{\text {nd }}$ Ed. New York: Rutledge, 2002.

Anales de Documentación, 2012, vol. 15, nº 1 


\section{ANEXO 1}

Encuesta sobre Cibercultura Estudiantil

Universidad Autónoma de Chihuahua

Facultad de Filosofía y Letras

ESTIMADA(O) ALUMNA(O):

Solicitamos tu amable colaboración para contestar este cuestionario con el que se pretende medir el grado de "culturización digital" de la comunidad estudiantil de la UACH- FFyL / ENAH-Chihuahua, como parte de un proyecto de investigación del Cuerpo Académico en Bibliotecología y Ciencias de la Información de la UACH.

La encuesta es de carácter anónimo y tus respuestas serán empleadas sólo con fines estadísticos, por lo cual te solicitamos contestes de una manera abierta y con la mayor veracidad posible. Responder este cuestionario te ocupará unos 10 minutos, por favor no dejes preguntas sin contestar, recuerda que lo importante para nosotros es tu opinión franca y completa.

INSTRUCCIONES:

Valora en los siguientes apartados, marcando con una $\mathrm{X}$, el grado o nivel de acuerdo con las frases que se te presentan, usando para ello la escala del 0 al 5:

\section{ACCESO A LAS TECNOLOGÍAS DE INFORMACIÓN Y COMUNICACIÓN}

\section{(TIC'S)}

Nivel de Acuerdo

Total Desacuerdo

Total Acuerdo

\begin{tabular}{|l|l|l|l|l|l|}
\hline 1.1. Tengo fácil acceso a la telefonía celular. & (1) & (2) & (3) & (4) & (5) \\
\hline 1.2. Tengo amplio acceso a Internet (Red global / WWW). & (1) & (2) & (3) & (4) & (5) \\
\hline $\begin{array}{l}\text { 1.3. Tengo acceso a una o varias intranets (Redes internas). } \\
\text { 1.4. La mayoría de mis conexiones a Internet son inalámbricas } \\
\text { o WiFi. }\end{array}$ & (1) & (2) & (3) & (4) & (5) \\
\hline
\end{tabular}
o WiFi.

\section{USO DE LAS TECNOLOGÍAS DE INFORMACIÓN Y COMUNICACIÓN}

\section{(TIC'S)}

Nivel de Acuerdo

Total Desacuerdo

Total Acuerdo

\begin{tabular}{|l|l|l|l|l|l|}
\hline $\begin{array}{l}\text { 2.1. Generalmente uso el celular para hacer o recibir llamadas } \\
\text { telefónicas y enviar mensajes de texto. }\end{array}$ & (1) & (3) & (4) & (5) \\
\hline $\begin{array}{l}\text { 2.2. También uso el celular para tomar fotos/videos, organizar } \\
\text { actividades, escuchar música, intercambiar datos, etc. }\end{array}$ & (1) & (2) & (3) & (4) & (5) \\
\hline $\begin{array}{l}\text { 2.3. Generalmente uso el Internet para enviar e-mails, chatear, } \\
\text { bajar música y videos, ver noticias, etc. }\end{array}$ & (2) & (3) & (4) & (5) \\
\hline
\end{tabular}




\section{DATOS GENERALES}

Carrera que estudias:

Semestre que cursas: ¿Cuál fue tu promedio escolar en el semestre anterior?:

Edad: __ Género: Masculino Femenino

Número de miembros en tu hogar:

Estado civil:

Trabajas actualmente: $\quad \mathrm{Si} \quad$ No

Colonia

donde

vives:

Número de focos en tu hogar:

¿Cuántas computadoras hay en tu casa?:

¿Tienes Internet en tu casa?: $\quad \mathrm{Si} \quad$ No

¿Cuántos teléfonos celulares hay en tu ¿Tienes laptop o PC portátil?: $\quad \mathrm{Si} \quad$ No casa?:

¿Cuántas horas al día te conectas a Internet?:

¿Dónde te conectas a Internet con más frecuencia?

¿Cuántas veces a la semana revisas tu mail? Escuela Hogar Cibercafé Oficina

¿Tienes reproductor digital de música, ¿Tienes tu propio blog?: $\quad$ Si $\quad$ No tipo Ipod, MP3 o similar?: Si No.

¿ALGÚN COMENTARIO PERSONAL?:

i GRACIAS POR TU PARTICIPACIÓN!!

Anales de Documentación, 2012, vol. 15, nº 1 